\title{
Comparable vascular response of a new generation sirolimus eluting stents when compared to fluoropolymer everolimus eluting stents in the porcine coronary restenosis model
}

Piotr P. Buszman ${ }^{1,2 *}$, Magdalena Michalak ${ }^{1}$, Maciej Pruski ${ }^{1}$, Carlos Fernande ${ }^{1}$, Michał Jelonek ${ }^{1}$, Adam Janas ${ }^{1}$, Claudine Savard ${ }^{3}$, Beata Gwiazdowska-Nowotka ${ }^{4}$, Aleksander Żurakowski ${ }^{1}$, Wojciech Wojakowski ${ }^{1,5}$, Paweł E. Buszman ${ }^{1,5}$, Krzysztof Milewski ${ }^{1}$

${ }^{1}$ Center for Cardiovascular Research and Development of American Heart of Poland, Katowice, Poland

${ }^{2}$ Silesian Center for Heart Diseases, Zabrze, Poland

${ }^{3}$ AccelLAB Inc., Boisbriand, Canada

${ }^{4}$ Balton, Warsaw, Poland

${ }^{5}$ Medical University of Silesia, Katowice, Poland

\begin{abstract}
Background: Novel sirolimus eluting stents (SES) have shown non-inferior clinical outcomes when compared to everolimus eluting stents (EES), however only limited preclinical data have been published. Therefore, we evaluate vascular response of a new generation biodegradable polymer SES (BP-SES: Alex Plus, Balton) and fluoropolymer EES (EES: Xience Pro, Abbott) in the porcine coronary restenosis model.

Methods: A total of 40 stents were implanted with $120 \%$ overstretch in coronaries of $17 \mathrm{dom}$ estic swine: 16 BP-SES, 16 EES and 8 bare metal controls (BMS). Following 28 and 90 days, coronary angiography and optical coherence tomography (OCT) was performed, animals sacrificed and stented segments harvested for pathological evaluation.

Results: At 28 days neointimal thickness in OCT was lowest in the BP-SES when compared to EES and BMS (0.18 \pm 0.1 vs. $0.39 \pm 0.1$ vs. $0.34 \pm 0.2 \mathrm{~mm}$, respectively; $p=0.04)$. There was no difference in the proportion of malapposed or uncovered struts, although protruding covered struts were more common in BP-SES (14.8 $\pm 10 \%$ vs. $4.1 \pm 4 \%$ vs. $3.7 \pm 6 \% ; p=0.03)$. In pathology, the lowest neointimal thickness was confirmed in BP-SES $(p<0.05)$. The inflammation score was significantly lower in BP-SES and EES when compared to BMS (0.24 \pm 0.1 vs. $0.4 \pm 0.1$ vs. $0.77 \pm 0.4 ; p<0.01)$ whilst EES and BP-SES had higher fibrin scores than BMS $(1.2 \pm 0.4$ vs. $1.3 \pm 0.3$ vs. $0.17 \pm 0.2 ; p<0.01)$. At 90 days neointimal coverage and thickness in OCT was comparable between groups and healing in histopathology was complete. Conclusions: New generation, BP-SES show similar vascular healing and biocompatibility profile with marginally higher degree of restenosis inhibition, when compared to fluoropolymer EES in the porcine coronary restenosis model. (Cardiol J 2016; 23, 6: 657-666)
\end{abstract}

Key words: everolimus eluting stent, new generation sirolimus eluting stent, biodegradable polymer, porcine coronary restenosis model

Address for correspondence: Piotr P. Buszman, MD, PhD, Center for Cardiovascular Research and Development, American Heart of Poland Inc, ul. Czajek 41, 40-534 Katowice, Poland 


\section{Introduction}

First generation sirolimus eluting stents (SES) although efficient in restenosis inhibition caused delayed healing and as a consequence increased restenosis and late thrombotic events when compared to second generation drug eluting stents (DES) [1-3]. It has been shown that autonomously sirolimus and its derivatives (everolimus, zotarolimus) have similar effects on vascular response and clinical outcomes $[4,5]$, therefore it has been postulated that the polymer and release kinetics were responsible for the negative tissue effect and clinical outcomes. The early generation SES consisted of non-erodible PEVA/PBMA polymers and thick strut profile stent platform which caused anaphylactic and inflammatory reactions, resulting in impaired vessel healing and ultimately adverse thrombotic events [6]. As a result, novel biodegradable polymers as well as low profile, cobalt or platinum chromium stent backbones were proposed, which resulted in improved vascular response [7]. Furthermore, sirolimus elution has been optimized to balance between safety and efficacy [8]. These technological refinements resulted in non-inferior clinical outcomes of second generation SES when compared to everolimus eluting stents (EES) in large randomized trials [5, 9-11]. However, the underlying temporal tissue vascular response, has been not well described. Therefore, in this study we evaluate in-vivo as well as histopathological temporal vascular healing and biocompatibility to second generation biodegradable polymer sirolimus eluting stents (BP-SES, Alex Plus, Balton) and compare it with EES (Xience Pro, Abbott) in the experimental setting of a porcine coronary instent restenosis model.

\section{Methods}

\section{Device description}

The studied SES (BP-SES, Alex Plus, Balton, Poland) employs fully biodegradable multilayer structure containing a copolymer of poly-lactic and glycolic acid and sirolimus at dose of $1.3 \mu \mathrm{g} /$ $/ \mathrm{mm}^{2}$ (data on file at Balton). The total mass of the polymer on a $3.0 \times 15 \mathrm{~mm}$ stent does not exceed $360 \mu \mathrm{g}$. The experimental studies in the porcine in-stent restenosis model at 8 weeks has shown nearly full polymer biodegradation and $95 \%$ drug release of initial drug load [12]. The stent utilizes the L605 cobalt chromium alloy platform with strut thickness of $70 \mu \mathrm{m}$ and closed cell design, which also served as bare metal control (BMS, Coflexus,
Balton). The cell area is larger by $50 \%$ when compared to predecessor (Alex, Balton, Poland) to facilitate easier side branch access.

Everolimus-eluting stent (Reference group, Xience Pro, Abbott, Santa Clara, USA) contains the drug at a dose of $100 \mathrm{mg} / \mathrm{cm}^{2}$ of stent surface and is coated with a fluoropolymer (poly(vinylidene fluoride-co-hexafluoropropylene) designed to release $80 \%$ of the everolimus in the first 30 days after deployment.

\section{Study design}

Study flow chart is presented in Figure 1. A total of 17 domestic swine of both genders were included. All animals ranged from 5 to 7 months of age with an average weight of around $45 \mathrm{~kg}$ at the time of enrolment. Middle arterial segments, without side branches of all three coronary arteries (RCA, LAD, LCX), screened for stent implantation. After qualitative coronary angiography (QCA) evaluation, 40 segments were eligible for the study and 40 stents, including 16 BP-SES (study group), 16 EES (reference group) and $8 \mathrm{BMS}$ controls were implanted after live QCA guidance to ensure $120 \%$ overstretch. Half of the animals were followed up for 28 days and the other half for 3 months. Subsequently control coronary angiography with optical coherence tomography (OCT) was performed and swine sacrificed. All stented segments were harvested for pathological analysis. All interventions and analyses were blinded to operators or investigators.

\section{Experimental procedures}

The study protocol was approved by the local ethics committee for animal research. All animals received standard care outlined in the study protocol and in accordance with the act of animal welfare and the "Principles of Care of Laboratory Animals" [13]. The experimental protocol has been published before [14]. Briefly, the animals were loaded with double anti-platelet therapy 3 days before intervention. Prior to procedure, swine were pre medicated with atropine $(0.5 \mathrm{mg})$ and subsequently sedated with intramuscular ketamine hydrochloride $(20 \mathrm{mg} / \mathrm{kg})$ and xylazine $(2 \mathrm{mg} / \mathrm{kg})$, intubated, and anesthetized with an intravenous propofol bolus (20-40 mg) followed by a continuous infusion $(2-4 \mathrm{mg} / \mathrm{kg} / \mathrm{h})$. A vascular sheath $(6 \mathrm{~F})$ was placed in the right or left femoral artery utilizing a Seldinger technique. Anticoagulation with heparin was achieved (3,000-10,000 IU) to maintain a coagulation time $\geq 250 \mathrm{~s}$. Following coronary angiography, all coronary vessels were 


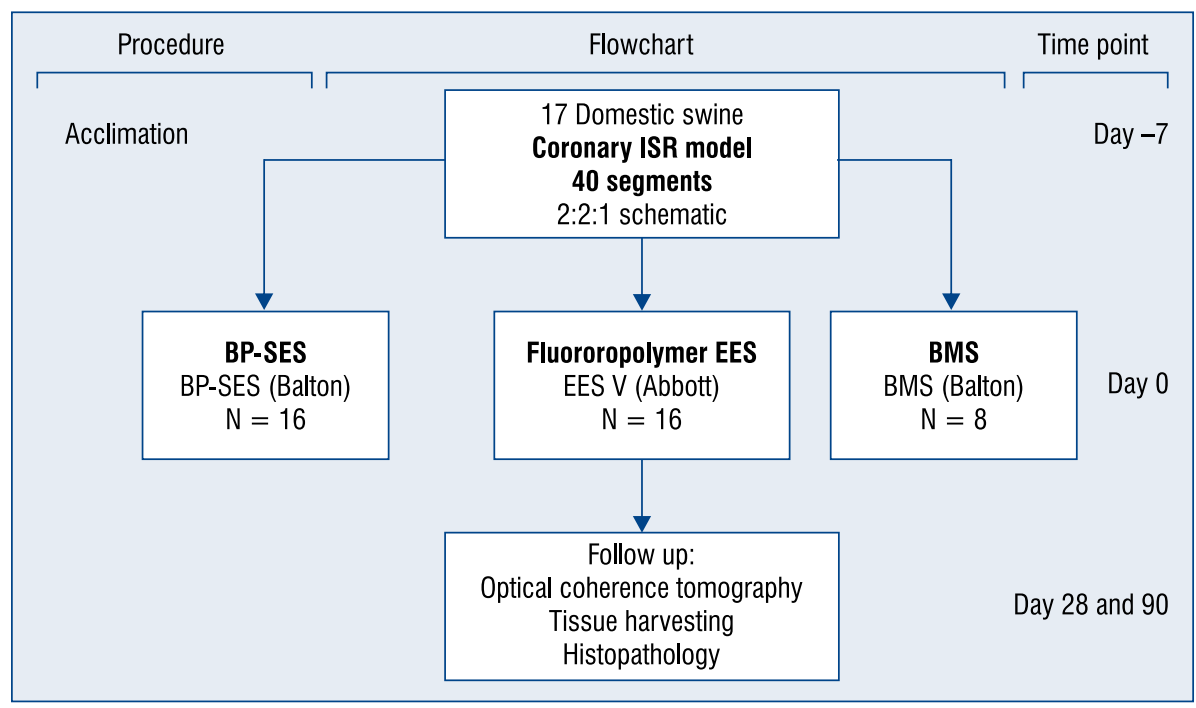

Figure 1. Study schematics; ISR — in-stent restenosis; BP-SES — biodegradable polymer sirolimus eluting stent; BMS — bare metal stent; EES — everolimus eluting stent.

sized for proper stent implantation after live QCA analysis. Stent were implanted with a pressure ensuring $120 \%$ arterial overstretch.

All pigs were anesthetized and prepared in the same fashion as described above at 28 days and 3 months following stent implantations to perform control coronary angiography and OCT. Subsequently animals were humanely sacrificed with pentobarbital overdose.

\section{Quantitative coronary analysis}

Coronary arteries angiographies were obtained using Siemens Coroskop Millenium Edition angiographic unit. Judkins Right, 6 French guiding catheter was utilized to obtain coronary angiography and stent implantation. QCA analysis was performed in a blinded fashion utilizing QAngio XA Software version 7.1.14.0 (Medis Medical Imaging Systems) from two contralateral projections. The baseline and 28-day follow-up reference vessel diameters (RVD) and minimal lumen diameters (MLD) were taken from the treated segments using the guiding catheter as a standard for measurement. The balloon-to-artery ratio was calculated. Percent diameter stenosis (\%DS) at follow-up was calculated as: $[1-(\mathrm{MLD} / \mathrm{RVD})] \times 100 \%$.

\section{Optical coherence tomography}

The OCT images of all tested coronary segments were acquired at 28 and 90 days follow up with a non-occlusive technique using the ILUMIEN $^{\mathrm{TM}}$ imaging system (St. Jude Medical, St.
Paul, MN). The OCT catheter was pulled back at $20 \mathrm{~mm} / \mathrm{s}$ and OCT images were generated at 100 frames/s. Contrast media (OMNIPAQUE 350; GE Healthcare) was continuously flushed through a guiding catheter during imaging catheter pullback to ensure blood clearance from the coronary arteries. Analysts who were blinded to procedural information analyzed all acquired images. Crosssectional OCT images were analyzed at $1 \mathrm{~mm}$ intervals. Reference vessel area was calculated at $5 \mathrm{~mm}$ distance from proximal and distal edge of stent. Neointimal thickness was calculated by subtracting the luminal cross-sectional areas from the stent. The strut appearance and coverage was qualified as embedded, protruding covered, protruding uncovered and malapposed according to previously described definitions $[15,16]$.

\section{Histological analysis}

Following vessel harvesting, stented segments were immersed in normal buffered formalin $10 \%$. Stented artery segments were embedded in methyl methacrylate, then divided into a target of 3 blocks identified as proximal, middle and distal. Thin sections (approximately $8 \mu \mathrm{m}$ ) from each stented artery block were stained with hematoxylin and eosin (H\&E) and Verhoeff-van Gieson (VVG). Images of each VVG-stained artery section (low magnification, whole section) were digitally captured. VVG-stained stented artery sections were examined by a trained operator for histomorphometry. All H\&E and VVG stained artery sections 
were examined by the study pathologist for semiquantitative and descriptive histopathology.

VVG-stained stented artery sections were examined using light microscopy, image capture, and quantitative morphometric computer-assisted methods with Image Pro Plus 6.1.0.346 software. For each section, the operator delineated the external elastic lamina (EEL - at the junction of the medial and adventitial layers), the internal elastic lamina (IEL - at the junction of the medial and neointimal layers), and the luminal border. Neointimal thickness was measured as the distance from the inner surface of the stent struts to the luminal border. The following measures were used to calculate vessel layer areas: Media = EEL-IEL; Neointima $=$ IEL-Lumen; \% Area Stenosis = $=[1-($ Lumen area $/$ IEL area $)] \times 100$.

Semi-quantitative microscopic evaluation of stented artery sections was performed. Scores were assigned to each stented section using the criteria listed below. Unstented sections were examined and significant observations were graded (when appropriate) and reported. All sections were evaluated using semi-quantitative scoring criteria. To evaluate the amount of injury, a criteria defined by Schwartz et al. [17] were utilized: $0=$ IEL intact, $1=$ IEL lacerated, $2=$ media lacerated, $3=$ EEL lacerated. To evaluate the extent of peri-strut inflammatory reaction the following grade by Kornowski et al. [18] was used: $0=$ minimal inflammatory response around strut, $1=$ few inflammatory cells around strut, $2=$ mild to moderate inflammation, can extend into but do not efface surrounding tissue, $3=$ dense and thick peri-strut aggregate of inflammatory cells, effacing surrounding tissue. Fibrin deposition was graded as follows (strut-by-strut): $0=$ absent, or rare minimal spotting around strut, 1 = fibrin in small amounts, localized only around strut, $2=$ fibrin moderately abundant or denser, extending beyond strut, 3 = abundant, dense fibrin, bridging between strut. Endothelialisation was assessed with regards to percentage coverage of artery circumference: $0=<25 \%, 1=25-75 \%, 2=76-99 \%, 3=$ complete endothelial coverage. Neointimal smooth muscle score representing neointimal maturity, estimates the proportion of neointimal areas containing smooth muscle cells: $0=$ no smooth muscle cells visible, $1=<25 \%, 2=25-75 \%, 3=>75 \%$ of neointima containing smooth muscle. Each strut in the section was scored and the mean inflammation and injury score for each section was calculated and reported.

\section{Statistical analysis}

Normally distributed parametric data are expressed as average and standard deviation, and as median and interquartile range $[\mathrm{IQR}]$ in cases of skewed distribution. When equal variance and normality were observed, one-way analysis of variance (ANOVA) with Student-Newman-Keuls post-ANOVA tests were used to test for differences in variables between stent types. When either equal variance test or normality test failed, Kruskal-Wallis test (with Dunn's method for post-hoc group comparison) was conducted. Tissue coverage was estimated through the average number of embedded, protruding covered, uncovered and mallaposed struts at a stent level. A value of $p \leq 0.05$ was considered statistically significant.

\section{Results}

\section{Coronary angiography}

There were no differences in baseline vessel diameters and overstretch ratios expressed as balloon to artery ratio in groups designated both for 30- and 90-day follow-up (Table 1). At 28 and 90 days all studied segments and vessels were patent, with no signs of thrombosis or in-stent restenosis. There were no differences in late lumen loss and percent diameter stenosis at 28 days. At 90 days the late lumen loss was significantly lowest in the BMS $(p=0.02)$ when compared to EES.

\section{Optical coherence tomography}

The OCT morphometrical results are presented in Figure 2. The stent areas were comparable between groups designated both for short and long-term follow up. At 28 days the neointimal thickness was significantly lowest in the BP-SES when compared to BMS and EES $(p<0.05)$. There was also a trend toward lowest percent area stenosis in the BP-SES when compared to BMS and EES $(p=0.082)$. At 3 months there were no differences in all OCT morphometric measurements between the groups.

The analysis of stent coverage and apposition is presented in Table 2. At 28 days follow up, there was a trend toward lower proportion of fully embedded struts and higher proportion of protruding, covered struts in the BP-SES when compared to BMS and EES $(p<0.05)$. There were no differences between uncovered and malapossed in any of the groups. At 90 days, $90 \%$ of struts in all groups were embedded and did not differ significantly between groups. The rate of uncovered stent struts was very low, and did not differ significantly between groups (Fig. 3). 
Table 1. Quantitative coronary angiography analysis at baseline, 28 and 90 day follow up.

\begin{tabular}{|c|c|c|c|c|}
\hline & BP-SES $(n=8)$ & BMS $(n=4)$ & EES $(n=8)$ & $\mathbf{P}$ \\
\hline \multicolumn{5}{|l|}{ Baseline } \\
\hline $\mathrm{RVD}[\mathrm{mm}]$ & $2.68 \pm 0.4$ & $2.56 \pm 0.4$ & $2.49 \pm 0.21$ & 0.54 \\
\hline$B-2-A$ & $1.2 \pm 0.1$ & $1.26 \pm 0.1$ & $1.28 \pm 0.1$ & 0.23 \\
\hline \multicolumn{5}{|l|}{28 days } \\
\hline $\mathrm{MLD}[\mathrm{mm}]$ & $2.46 \pm 0.4$ & $2.28 \pm 0.2$ & $2.30 \pm 0.5$ & 0.6 \\
\hline$\%$ DS & $9.85 \pm 3.7$ & $8.86 \pm 2.4$ & $10.68 \pm 5.8$ & 0.8 \\
\hline LL [mm] & $0.25 \pm 0.2$ & $0.49 \pm 0.3$ & $0.36 \pm 0.3$ & 0.3 \\
\hline \multicolumn{5}{|l|}{90 days } \\
\hline $\mathrm{MLD}[\mathrm{mm}]$ & $2.48 \pm 0.4$ & $2.56 \pm 0.3$ & $2.13 \pm 0.6$ & 0.2 \\
\hline$\%$ DS & $10.5 \pm 11.2$ & $8.8 \pm 3.3$ & $22.3 \pm 15$ & 0.07 \\
\hline $\mathrm{LL}[\mathrm{mm}]$ & $0.3 \pm 0.23$ & $0.1 \pm 0.1^{*}$ & $0.76 \pm 0.5$ & 0.02 \\
\hline
\end{tabular}

${ }^{*} p<0.05$ vs. Xience; B-2-A — balloon to artery ratio; BP-SES — biodegradable polymer sirolimus eluting stent; BMS — bare metal stent; $\% D S$ - percent diameter stenosis; EES — everolimus eluting stent; LL — late lumen loss; MLD — minimal lumen diameter; RVD — reference vessel diameter

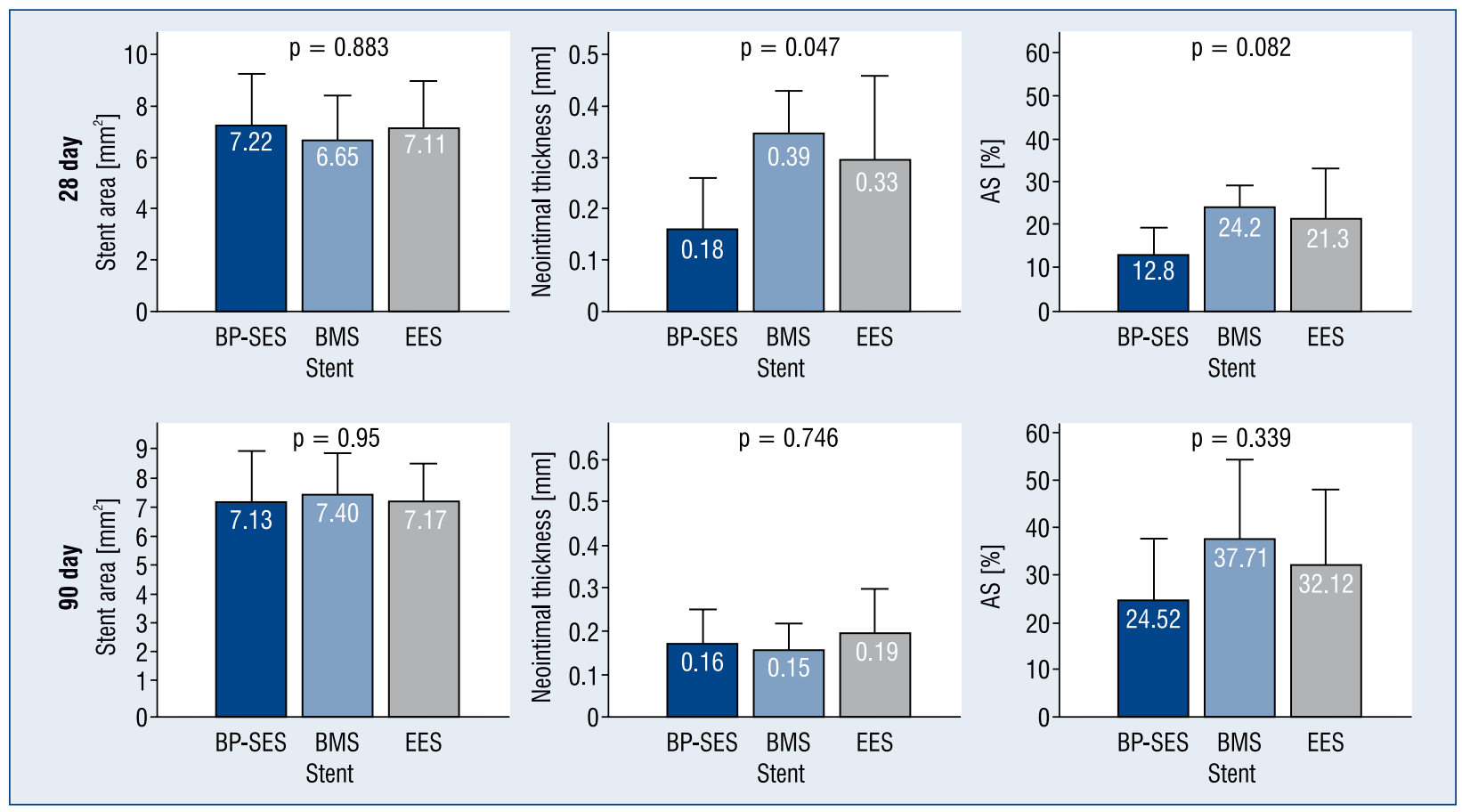

Figure 2. Optical coherence tomography evaluation depicting morphometric analysis of neointimal thickness, stent area and percent area stenosis (AS) at 28 (upper panel) and 90 day follow-up (lower panel); BP-SES - biodegradable polymer sirolimus eluting stent; BMS - bare metal stent; EES — everolimus eluting stent.

\section{Histopathological evaluation}

A detailed histopathological analysis is presented in the Tables 3 and 4 . In all animals the vessel sizes were comparable as represented by the IEL and EEL areas. At 28 days, all vessels were patent with no binary in-stent restenosis or thrombus. The percent area stenosis was lowest in the BP-SES group $(\mathrm{p}<0.05$ when compared to BMS). Correspondingly, the mean neointimal thickness was lowest in the BP-SES ( $p<0.05$ when compared both to EES and BMS). Endothelialisation and neointimal maturity expressed as smooth muscle cell score were comparable, however the inflammation score was significantly lower in BP-SES and EES when compared to BMS by $70 \%$ 
Table 2. Optical coherence tomography stent and vessel morphometry analysis at 28 and 90 days follow up.

\begin{tabular}{|c|c|c|c|c|}
\hline Per-stent analysis & BP-SES $(n=8)(\%)$ & EES $(n=8)(\%)$ & BMS $(n=4)(\%)$ & $\mathbf{P}$ \\
\hline \multicolumn{5}{|l|}{28 days } \\
\hline Embedded & $78.2 \pm 24$ & $95.4 \pm 7$ & $94.2 \pm 4$ & 0.11 \\
\hline Protruding covered & $14.8 \pm 10^{*}$ & $4.1 \pm 4$ & $3.7 \pm 6$ & 0.03 \\
\hline Protruding uncovered & $6.8 \pm 15.7$ & $1.5 \pm 3$ & $0.7 \pm 1$ & 0.51 \\
\hline Malapposed & $0.1 \pm 0.3$ & $0.1 \pm 0.2$ & 0 & 0.78 \\
\hline \multicolumn{5}{|l|}{90 days } \\
\hline Embedded & $86.7 \pm 18$ & $85.1 \pm 20$ & $91.3 \pm 8$ & 0.75 \\
\hline Protruding covered & $11.2 \pm 16$ & $11.3 \pm 15$ & $7.9 \pm 8$ & 0.86 \\
\hline Protruding uncovered & $1.95 \pm 3$ & $3.7 \pm 6$ & 0 & 0.33 \\
\hline Malapposed & $0.1 \pm 0.2$ & 0 & 0 & 0.59 \\
\hline
\end{tabular}

${ }^{*} \mathrm{p}=0.05$ vs. Xience; BP-SES - biodegradable polymer sirolimus eluting stent; BMS — bare metal stent; EES — everolimus eluting stent

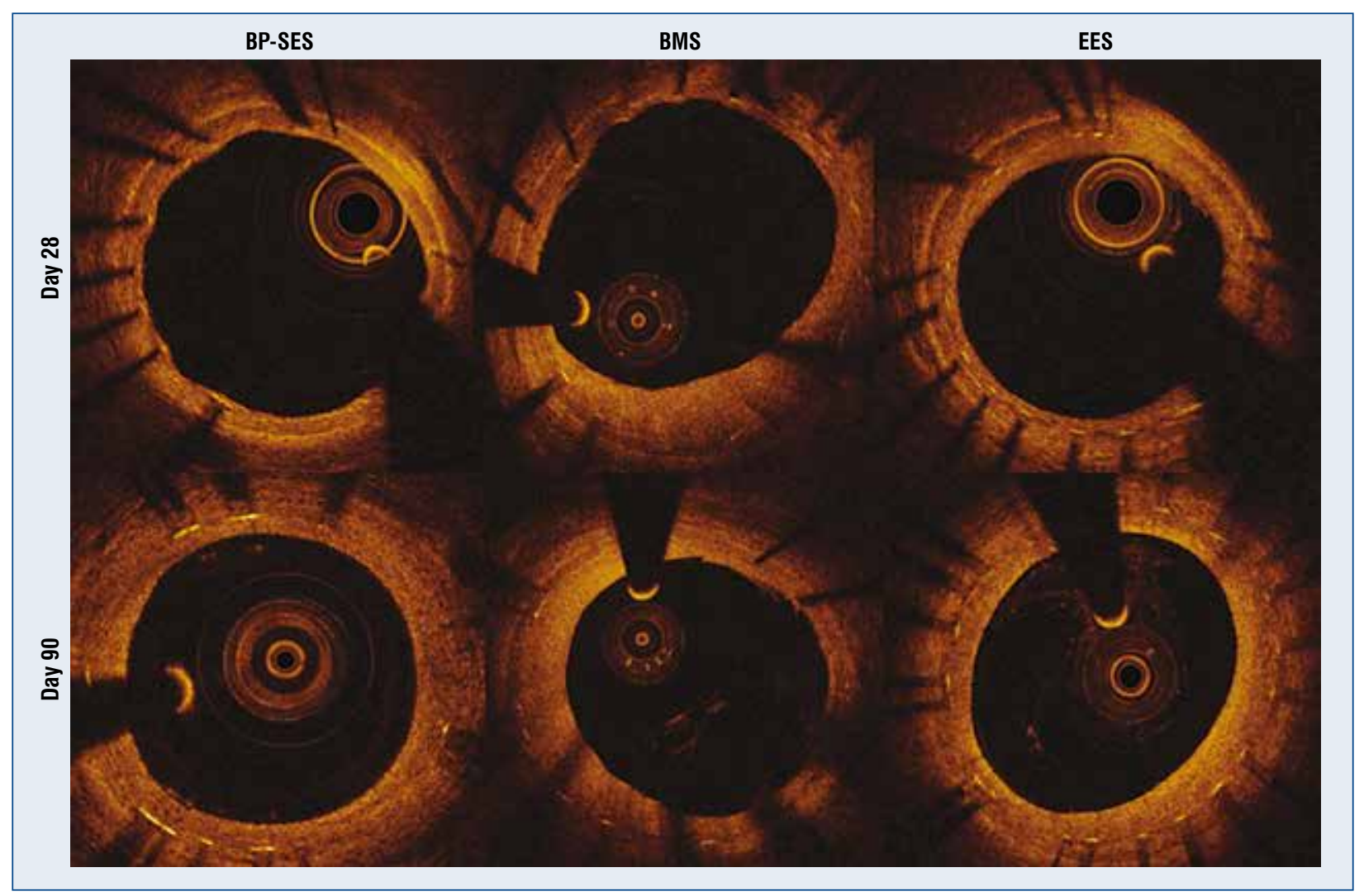

Figure 3. Optical coherence tomography. Representative stent cross sections; BP-SES — biodegradable polymer sirolimus eluting stent; BMS - bare metal stent; EES - everolimus eluting stent.

and $40 \%$, respectively. On the other hand, the fibrin deposition was nearly absent in BMS and reported in both DES $(p<0.05)$. The key findings of the histopathological analysis are presented in Figure 4, whereas the representative peri-strut magnification and stent cross section photomicrographs are shown in Figure 5.

At 90 days neointimal hyperplasia expressed as percent area stenosis and neointimal thickness was comparable among groups. The healing was 
Table 3. Histopathology at 28 days.

\begin{tabular}{|c|c|c|c|c|c|}
\hline \multirow[t]{2}{*}{ Parameters } & \multirow{2}{*}{$\begin{array}{l}\text { BP-SES } \\
(\mathrm{n}=8)\end{array}$} & \multirow{2}{*}{$\begin{array}{l}\text { BMS } \\
(n=4)\end{array}$} & \multirow{2}{*}{$\begin{array}{c}\text { EES } \\
(n=8)\end{array}$} & \multicolumn{2}{|r|}{ One-way ANOVA } \\
\hline & & & & $\mathbf{P}$ & Post hoc \\
\hline \multicolumn{6}{|l|}{ Histomorphometry } \\
\hline EEL area $\left[\mathrm{mm}^{2}\right]$ & $8.64 \pm 2.09$ & $8.77 \pm 1.78$ & $8.82 \pm 1.93$ & 0.941 & - \\
\hline IEL area $\left[\mathrm{mm}^{2}\right]$ & $7.39 \pm 1.81$ & $7.30 \pm 1.46$ & $7.46 \pm 1.58$ & 0.928 & - \\
\hline Medial area $\left[\mathrm{mm}^{2}\right]$ & $1.25 \pm 0.29$ & $1.47 \pm 0.38$ & $1.36 \pm 0.37$ & 0.578 & - \\
\hline Neointimal area $\left[\mathrm{mm}^{2}\right]$ & $0.98 \pm 0.33$ & $2.27 \pm 0.44$ & $1.56 \pm 0.55$ & $<0.001$ & $\begin{array}{l}<0.001: \text { BP-SES vs. BMS } \\
\text { 0.038: BP-SES vs. EES } \\
\text { < 0.050: EES vs. BMS }\end{array}$ \\
\hline Luminal area $\left[\mathrm{mm}^{2}\right]$ & $6.42 \pm 1.93$ & $5.03 \pm 1.35$ & $5.90 \pm 1.68$ & 0.447 & - \\
\hline Area stenosis [\%] & $14.1 \pm 6.7$ & $31.4 \pm 6.8$ & $21.7 \pm 9.5$ & 0.014 & $<0.05$ : BP-SES vs. BMS \\
\hline Mean intimal thickness [mm] & $0.11 \pm 0.04$ & $0.26 \pm 0.06$ & $0.17 \pm 0.07$ & 0.002 & $\begin{array}{c}<0.001 \text { : BP-SES vs. BMS } \\
<0.050: \text { EES vs. BMS }\end{array}$ \\
\hline \multicolumn{6}{|l|}{ Healing and biocompatibility } \\
\hline Injury score & $0.21 \pm 0.13$ & $0.26 \pm 0.14$ & $0.24 \pm 0.29$ & 0.687 & - \\
\hline Inflammation score & $0.24 \pm 0.12$ & $0.77 \pm 0.40$ & $0.40 \pm 0.14$ & 0.002 & $\begin{array}{l}\text { 0.002: BP-SES vs. BMS } \\
0.022: \text { EES vs. BMS }\end{array}$ \\
\hline Fibrin score & $1.18 \pm 0.43$ & $0.17 \pm 0.22$ & $1.34 \pm 0.26$ & $<0.001$ & $\begin{array}{l}<0.001: \text { BP-SES } \\
\text { and EES vs. BMS }\end{array}$ \\
\hline Endothelialisation score & $2.21 \pm 0.39$ & $1.83 \pm 0.34$ & $2.17 \pm 0.64$ & 0.463 & - \\
\hline Neointimal smooth muscle score & $2.00 \pm 0.00$ & $2.00 \pm 0.27$ & $2.00 \pm 0.25$ & 1.000 & - \\
\hline
\end{tabular}

BMS — bare metal stent; BP-SES - biodegradable polymer sirolimus eluting stent; EES — everolimus eluting stent; EEL - external elastic lamina; IEL - internal elastic lamina

Table 4. Histopathology at 90 days.

\begin{tabular}{|c|c|c|c|c|}
\hline Parameters & $\begin{array}{l}\text { BP-SES } \\
(n=8)\end{array}$ & $\begin{array}{c}\text { BMS } \\
(n=4)\end{array}$ & $\begin{array}{c}\text { EES } \\
(n=8)\end{array}$ & $\begin{array}{l}\text { One-way } \\
\text { ANOVA: p }\end{array}$ \\
\hline \multicolumn{5}{|l|}{ Histomorphometry } \\
\hline $\mathrm{EEL}$ area $\left[\mathrm{mm}^{2}\right]$ & $8.14 \pm 3.31$ & $7.44 \pm 1.93$ & $8.23 \pm 1.43$ & 0.862 \\
\hline IEL area $\left[\mathrm{mm}^{2}\right]$ & $6.10 \pm 1.94$ & $6.29 \pm 1.77$ & $7.03 \pm 1.31$ & 0.530 \\
\hline Medial area $\left[\mathrm{mm}^{2}\right]$ & $1.87 \pm 1.76$ & $1.15 \pm 0.23$ & $1.17 \pm 0.22$ & 0.678 \\
\hline Intimal area $\left[\mathrm{mm}^{2}\right]$ & $2.36 \pm 0.81$ & $2.78 \pm 1.35$ & $2.88 \pm 1.51$ & 0.691 \\
\hline Luminal area $\left[\mathrm{mm}^{2}\right]$ & $3.69 \pm 1.72$ & $3.47 \pm 1.32$ & $4.12 \pm 1.67$ & 0.776 \\
\hline Area stenosis [\%] & $40.2 \pm 11.8$ & $44.2 \pm 15.1$ & $41.1 \pm 21.2$ & 0.927 \\
\hline Mean intimal thickness [mm] & $0.29 \pm 0.11$ & $0.35 \pm 0.15$ & $0.36 \pm 0.23$ & 0.803 \\
\hline \multicolumn{5}{|l|}{ Healing and biocompatibility } \\
\hline Injury score & $0.60 \pm 0.70$ & $0.78 \pm 0.57$ & $0.62 \pm 0.66$ & 0.745 \\
\hline Inflammation score & $1.04 \pm 0.83$ & $1.34 \pm 0.88$ & $1.13 \pm 0.88$ & 0.656 \\
\hline Fibrin score & $0.23 \pm 0.18$ & $0.26 \pm 0.17$ & $0.26 \pm 0.14$ & 0.930 \\
\hline Endothelialization score & $1.79 \pm 0.40$ & $2.17 \pm 0.34$ & $2.11 \pm 0.43$ & 0.265 \\
\hline Neointimal smooth muscle score & $1.58 \pm 0.39$ & $1.75 \pm 0.42$ & $1.54 \pm 0.47$ & 0.813 \\
\hline
\end{tabular}

BMS - bare metal stent; BP-SES — biodegradable polymer sirolimus eluting stent; EES — everolimus eluting stent; EEL - external elastic lamina; IEL - internal elastic lamina

nearly complete and comparable between stents (Table 4). The key histological parameters are highlighted in Supplementary Figure 1 (see journal website), whilst representative high resolution photomicrographs in Supplementary Figure 2 (see journal website). 


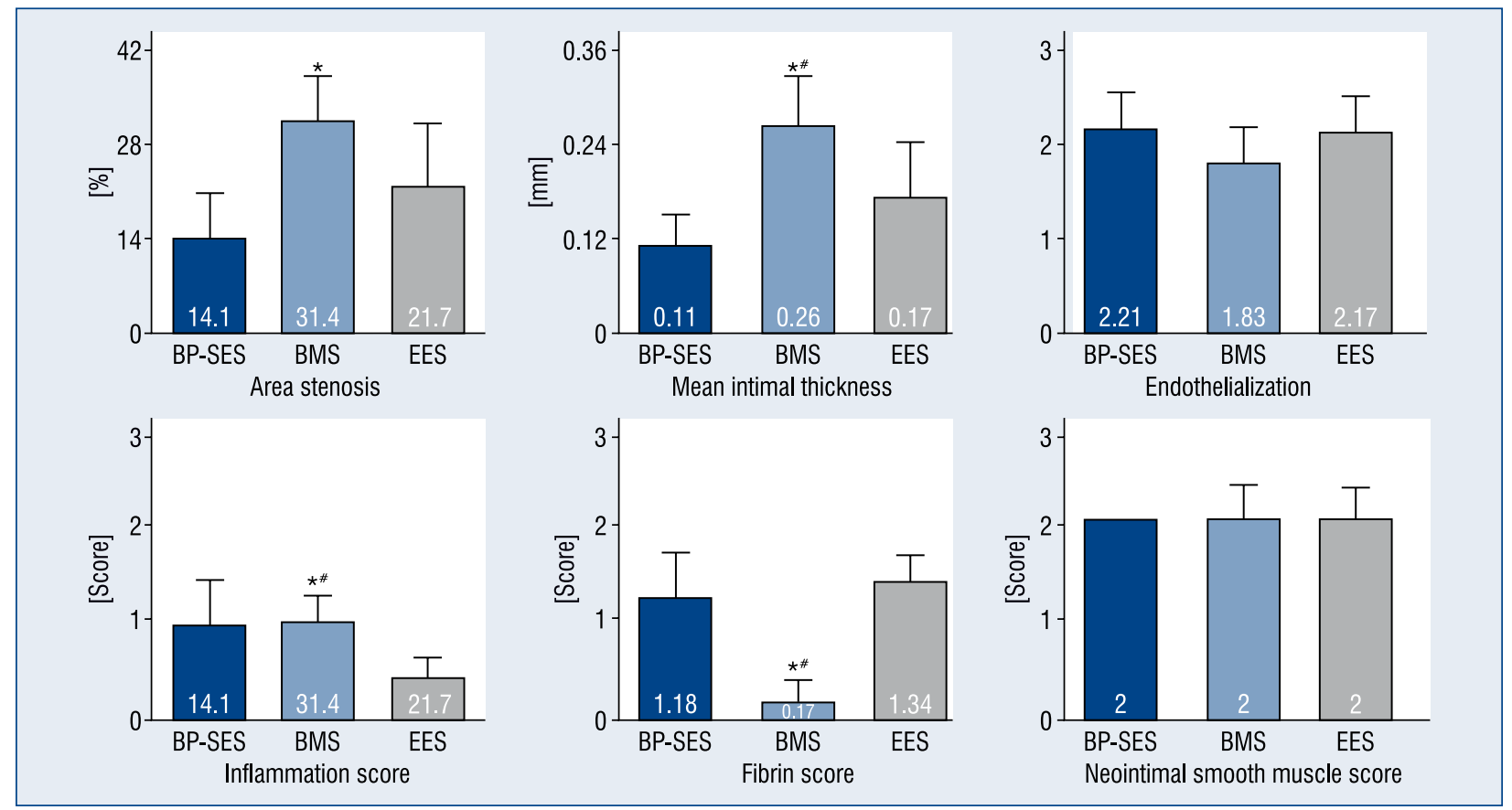

Figure 4. Bar charts featuring histopathological analysis of healing and biocompatibility at 28 day follow-up; BP-SES biodegradable polymer sirolimus eluting stent; BMS - bare metal stent; EES - everolimus eluting stent; ${ }^{*} p<0.05$ : BP-SES vs. BMS; \#p < 0.050: EES vs. BMS.

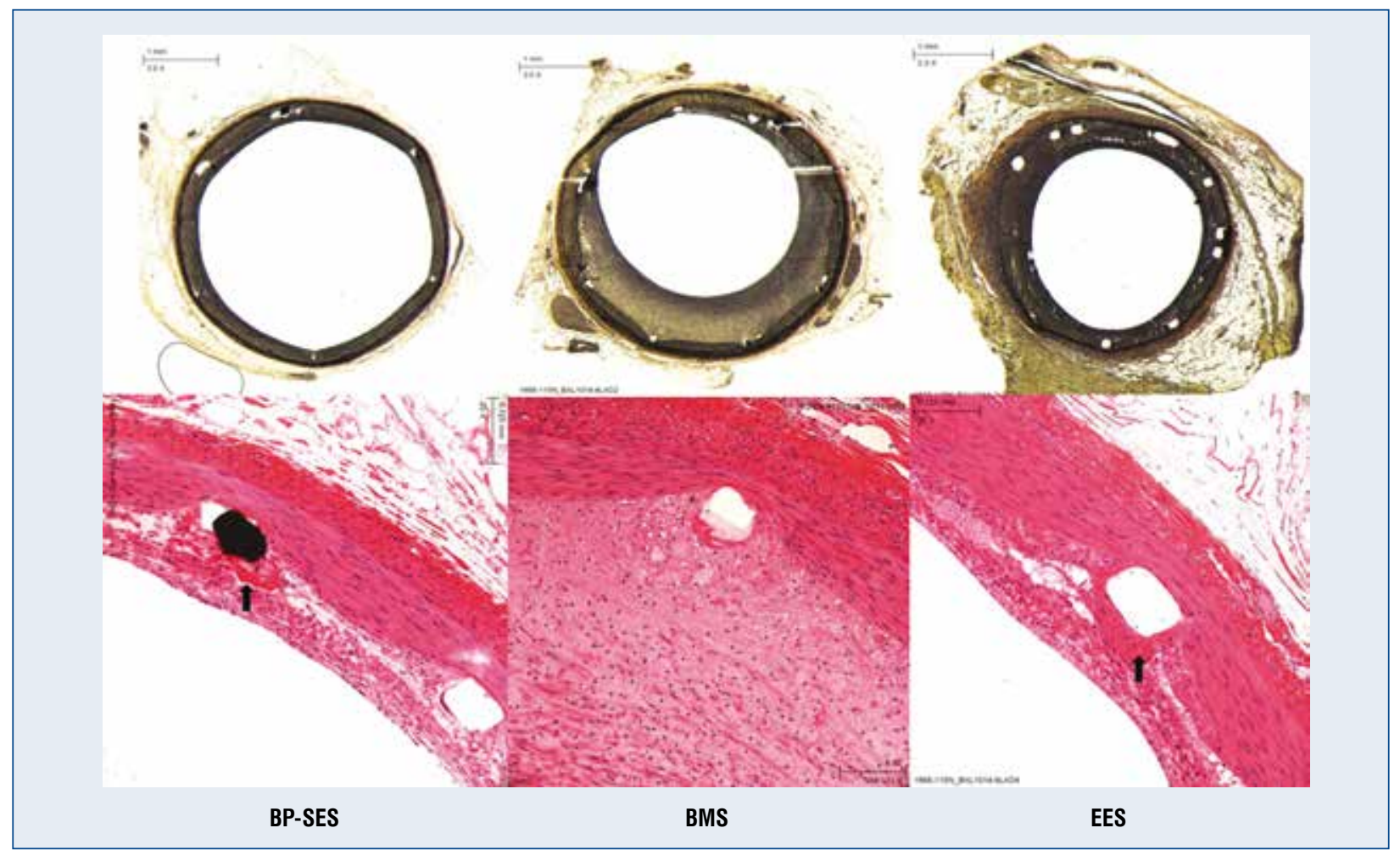

Figure 5. Representative high power images of peri-strut magnifications $(20 \times)$ stained with hematoxylin and eosin (lower panel) and stent cross sections stained with Verhoeff-van Gieson (VVG) at 28 day follow-up (upper panel). Fibrin depositis (arrow) visible in BP-SES and EES whereas higher neointimal formation and inflammatory response visible in BMS; BP-SES - biodegradable polymer sirolimus eluting stent; BMS — bare metal stent; EES — everolimus eluting stent. 


\section{Discussion}

In current study we evaluate in-vivo and histopathological vascular response to the novel, BP-SES (Alex Plus, Balton) and compare with the fluoropolymer everolimus eluting (Xience Pro, Abbott) stent in the porcine coronary in-stent restenosis model at short and mid-term follow-up. At 28 days BP-SES provided superior neointimal hyperplasia inhibition when compared to BMS. Additionally, there was a tendency toward lower neointimal thickness in OCT in BP-SES when compared to EES. On the other hand, there was a tendency toward improved neointimal coverage in EES in OCT. Angiographically, the late lumen loss was lowest in BMS, however we believe this is an incidental fining, most likely due to low number of BMS, low resolution of angiography and reassuring results in OCT analysis. In pathology at one month, the healing was comparable between EES and BPSES and most interestingly the inflammation was lowest in both DES when compared to BMS. Otherwise, the fibrin deposition was increased in both new generation DES. At 90 days the in-vivo and pathological exponents of healing and biocompatibility were comparable and complete in all groups, thus confirming the safety of a studied BP-SES. Endothelialization was incomplete in all groups, but these results were suspected to be related to pre-terminal injury to the endothelium by devices used for procedures such as OCT, and not to a real delay in endothelialisation process. This notion is supported by the fact that full endothelialisation of the studied BP-SES at 28 days has been shown before [19].

Previously, the first generation, durable polymer SES (Cypher, Cordis) showed inferior vascular response when compared to new generation DES [20] which has later translated into inferior clinical outcome. Therefore, to the best of our knowledge this is the first study which evaluated the preclinical in-vivo and vascular tissue response to a novel generation SES and directly compared the healing with current golden standard, the everolimus eluting fluropolymer EES (Xience Pro). This is important in translation to already published clinical trials of similar technologies, which have shown non-inferior clinical and angiographic outcomes between novel generation SES and fluropolymer EES with regard to the incidence of major adverse cardiac events $[5,10,21]$.

In current study, the OCT analysis of the vascular response at 28 days, showed that the neointimial thickness was smaller in BP-SES. We can hypothesize that except from the drug released this could be due to different drug release kinetics, which in BP-SES is faster when compared to EES.

The strut coverage in OCT, was slightly inferior in BP-SES when compared to fluropolymer EES. Importantly this was not associated with impaired healing in the histopathological evaluation. The clinical translation of impaired strut coverage in OCT is unclear, however up-to date the decreased proportion of protruding but covered struts has not translated into poorer outcome in the clinical setting [10]. The tissue coverage of currently evaluated BP-SES (Alex Plus) has been also assessed in the setting of a prospective clinical registry, with OCT evaluation at different time points [22]. At 3 month follow up the proportion of embedded struts was $85.8 \%$ and uncovered $10.3 \%$ whereas at 6 -months the number of uncovered struts was only $1.5 \%$ at 3 months. These proportions resemble current experimental observations and validate this pre-clinical model.

Both DES showed increased fibrin deposition when compared to BMS, which is considered a fingerprint of drug elution and retention in tissue and has been reported previously [4, 23]. The inflammatory reaction to implanted stents in this experiment deserves attention and discussion. At 1 month follow up, the pathological evaluation has reported significantly lower inflammation in both new generation DES when compared to BMS. This has been reported earlier with first generation SES [23], however at 3 months the inflammation tended to increase, which is contrary to our findings with novel generation SES and no inflammation at terminal follow-up. This preferable biocompatibility profile of tested EES and SES with very low peristrut inflammation at each study point, is supported by previously published reports suggesting that stent-based delivery of these analogues selectively decreases inflammation by clearing macrophages by autophagy, an mTOR inhibition-dependent mechanism inducing cell death in mammalian cells [24]. We believe that lower inflammation caused by modern DES is responsible for improved healing and lower late and very late stent thrombosis when compared to DES [25].

\section{Limitations of the study}

The limitations of this study include the nature of an experimental preclinical model as a human clinical surrogate and utilization of a healthy domestic swine, without underlying disease. 


\section{Conclusions}

In this experimental study, new generation, biodegradable polymer SES yielded similar healing and biocompatibility profile, when compared to fluoropolymer EES, whereas the inhibition of neointimal hyperplasia was in favour for BP-SES. The slightly impaired in-vivo stent coverage in OCT did not translate into poorer healing and biocompatibility in the pathological evaluation. Furthermore, both DES showed lower degree of inflammation when compared bare metal stents, thus supporting the unrestricted use of this technology in the clinical setting.

Funding: This study was funded by the Balton Ltd, Warsaw with the unrestricted reserch grant.

Conflict of interest: Piotr Buszman and Krzysztof Milewski - Research Contracts, Speakers Bureau, Balton; Beata Gwiazdowska-Nowotka is a full time employe at Balton; Sponsor's employee and representative.

\section{References}

1. Kubo T, Akasaka T, Kozuma Ket al. Comparison of neointimal coverage between everolimus-eluting stents and sirolimus-eluting stents: an optical coherence tomography substudy of the RESET (Randomized Evaluation of Sirolimus-eluting versus Everolimuseluting stent Trial). EuroIntervention, 2015; 11: 564-571.

2. Barbash IM, Minha S, Torguson R et al. Long-term safety and efficacy of the everolimus-eluting stent compared to first-generation drug-eluting stents in contemporary clinical practice. J Invasive Cardiol, 2014; 26: 154-160.

3. Waksman R, Barbash IM, Dvir D et al. Safety and efficacy of the XIENCE V everolimus-eluting stent compared to first-generation drug-eluting stents in contemporary clinical practice. Am J Cardiol, 2012; 109: 1288-1294.

4. Steigerwald K, Ballke S, Quee SC et al. Vascular healing in drug-eluting stents: differential drug-associated response of limus-eluting stents in a preclinical model of stent implantation. EuroIntervention, 2012; 8: 752-759.

5. Saito S, Valdes-Chavarri M, Richardt G et al. A randomized, prospective, intercontinental evaluation of a bioresorbable polymer sirolimus-eluting coronary stent system: the CENTURY II (Clinical Evaluation of New Terumo Drug-Eluting Coronary Stent System in the Treatment of Patients with Coronary Artery Disease) trial. Eur Heart J, 2014; 35: 2021-2031.

6. van der Giessen WJ, Lincoff AM, Schwartz RS et al. Marked inflammatory sequelae to implantation of biodegradable and nonbiodegradable polymers in porcine coronary arteries. Circulation, 1996; 94: 1690-1697.

7. Koppara T, Wittchow E, Byrne RA, Bayer G, Diener T, Joner M. Permanent and biodegradable polymer coatings in the absence of antiproliferative drugs in a porcine model of coronary artery stenting. EuroIntervention, 2016; 11: 1020-1026.

8. Stefanini GG, Holmes DR, Jr. Drug-eluting coronary-artery stents. N Engl J Med, 2013; 368: 254-265.
9. Christiansen EH, Jensen LO, Thayssen P et al. Scandinavian Organization for Randomized Trials with Clinical Outcome Vi. Biolimus-eluting biodegradable polymer-coated stent versus durable polymer-coated sirolimus-eluting stent in unselected patients receiving percutaneous coronary intervention (SORT OUT V): A randomised non-inferiority trial. Lancet, 2013; 381: 661-669.

10. Kimura T, Morimoto T, Natsuaki M et al., Investigators R. Comparison of everolimus-eluting and sirolimus-eluting coronary stents: 1-year outcomes from the Randomized Evaluation of Sirolimus-eluting Versus Everolimus-eluting stent Trial (RESET). Circulation, 2012; 126: 1225-1236.

11. Smits PC, Hofma S, Togni $\mathrm{M}$ et al. Abluminal biodegradable polymer biolimus-eluting stent versus durable polymer everolimus-eluting stent (COMPARE II): A randomised, controlled, non-inferiority trial. Lancet, 2013; 381: 651-660.

12. Orlik B, Buszman PP, Krauze A et al. A nuclear magnetic resonance spectroscopy as a method for evaluation of in vivo poly-1-lactide biodegradation kinetics from stent-polymer matrices: an experimental study utilizing porcine model of in-stent restenosis. J Cardiovasc Pharmacol Ther, 2016; 21: 93-99.

13. Institute of Laboratory Animal Resources NRC. Principles of Care of Laboratory Animals. NIH Publication, 1996; No. 85-23.

14. Buszman PP, Milewski K, Pajak J et al. Differences in vessel healing following delivery of everolimus or paclitaxel: a comparative experimental study using identical stent and biodegradable polymer platforms. EuroIntervention, 2014; 10: 724-731.

15. Lowe HC, Narula J, Fujimoto JG, Jang IK. Intracoronary optical diagnostics current status, limitations, and potential. JACC Cardiovasc Interv, 2011; 4: 1257-1270.

16. Bezerra HG, Costa MA, Guagliumi G, Rollins AM, Simon DI. Intracoronary optical coherence tomography: A comprehensive review clinical and research applications. JACC Cardiovasc Interv, 2009; 2: 1035-1046.

17. Schwartz RS, Huber KC, Murphy JG et al. Restenosis and the proportional neointimal response to coronary artery injury: Results in a porcine model. J Am Coll Cardiol, 1992; 19: 267-274.

18. Kornowski R, Hong MK, Tio FO, Bramwell O, Wu H, Leon MB. In-stent restenosis: Contributions of inflammatory responses and arterial injury to neointimal hyperplasia. J Am Coll Cardiol, 1998; 31: 224-230.

19. Buszman PP, Orlik B, Pajak J et al. Dose-dependent vascular response following delivery of sirolimus via fast releasing, biodegradable polymer stent matrix: an experimental study in the porcine coronary model of restenosis. Kardiol Pol, 2015; 73: 916-923.

20. Habib A, Karmali V, John MC et al. Everolimus-eluting stents improve vascular response in a diabetic animal model. Circ Cardiovasc Interv, 2014; 7: 526-532.

21. Pilgrim T, Heg D, Roffi M et al. Ultrathin strut biodegradable polymer sirolimus-eluting stent versus durable polymer everolimus-eluting stent for percutaneous coronary revascularisation (BIOSCIENCE): A randomised, single-blind, non-inferiority trial. Lancet, 2014; 384: 2111-2122.

22. Legutko JG, Robert J, Buszman PE et al. TCT-565 OCT evaluation of the time course of vessel healing following implantation of new generation biodegradable polymer-coated and sirolimuseluting cobalt-chromium coronary stent system (ALEX OCT Study). J Am Coll Cardiol, 2013; 62: B170-B171.

23. Nakazawa G, Finn AV, John MC, Kolodgie FD, Virmani R. The significance of preclinical evaluation of sirolimus-, paclitaxel-, and zotarolimus-eluting stents. Am J Cardiol, 2007; 100: 36M-44M.

24. Verheye S, Martinet W, Kockx MM et al. Selective clearance of macrophages in atherosclerotic plaques by autophagy. J Am Coll Cardiol, 2007; 49: 706-715.

25. Bonaa KH, Mannsverk J, Wiseth R et al. Drug-eluting or baremetal stents for coronary artery disease. N Engl J Med, 2016; 375: $1242-1252$. 